\title{
EDITORIAL
}

\section{Portable Sleep Studies for Diagnosis of Sleep Apnea - Where We are Today?}

\section{J. C. Suri}

See end of article for authors

Indian J Sleep Med 2006; 1.2, 65-71 affiliations

Address for correspondence:

J. C. Suri, Head of the

Department, Department of

Pulmonary, Critical Care \&

Sleep Medicine, Vardhaman

Mahavir Medical College \&

Safdarjang Hospital, New Delhi

jcsuri@del3.vsnl.net.in

$P^{b}$ eep D isordered Breathing (SD B) is increasingly being recognized as an important cause of morbidity and mortality. Ever since the first report of obstructive sleep apnea (OSA) by Guilliminault et al (1) there has been a tremendous growth in scientific literature related to various aspects of OSA. According to the Terry Young's data derived from the W isconsin Sleep C ohort Study (2), the prevalence of asymptomatic sleep apnea with an apnea - hyponea index (AH I) of - $\geq 5$ was $24 \%$ in men and $9 \%$ in women aged 30-60 yrs and that of symptomatic sleep apnea (i.e. $\mathrm{AHI}$ of $\geq 5$ with excessive daytime sleepiness) for men and women was $4 \%$ and $2 \%$ respectively. Untreated OSA is frequently associated with decrease in cognitive function (3), impairment of psychomotor function (4) and low levels of alertness leading to increased risk of vehicular and work-related accidents $(5,6)$. O SA is also associated with increased cardiovascular morbidity due to systemic and pulmonary hypertension (7-10), cardiac arrhythmia (11) and ischemic heart disease $(12,13)$. Studies have shown a reduced life expectancy and increased mortality of OSA patients from cardiovascular causes $(14,15)$ and stroke (16). The cumulative eight-year mortality of untreated OSA has been estimated as high as 37\% for patients with an $\mathrm{AHI}>20$ (14). In the W isconsin Sleep cohort Study (2), Young et al reported that $93 \%$ of females and $82 \%$ of males with moderate to severe sleep apnea remained undiagnosed. Because of its high prevalence, morbidity, mortality and public safety risks in untreated patients, OSA has been described as a major public health problem $(17,18)$. A number of placebo-controlled studies have shown that treatment of severe sleep apnea (i.e., $\mathrm{AHI} \geq 30$ ) with nasal continuous positive airway pressure (nCPAP) (19) improves alertness (20), decreases accident rates (21) and lowers blood pressure levels (22). H owever, data for treating mild to moderate OSA (i.e., $\mathrm{AH}$ I between $5 \& 30$ ) is less convincing (23-25). H ence identifying and then treating severe OSA in the community are of utmost importance. Unfortunately, there is a poor awareness of symptoms and signs of SD B in the community and wide variance of knowledge amongst medical practitioners (26). This makes a strong case for screening general population and more importantly specific high risk group for OSA.

The gold standard for the diagnosis of OSA is overnight in-laboratory technician attended polysomnography (PSG). A standard PSG consists of at least two channels of electroencephalogram (EEG), submental and tibial electromyogram (EMG), two channel s of electrooculogram (EOG), airflow, respiratory effort (thoracic and abdominal movements), oxygen saturation (oximetry) and electrocardiography (ECG). In addition there is a channel for body position and 
snoring (microphone) (27). PSG is time consuming, expensive, labour-intensive, and requires considerable technical expertise to perform and interpret (27). There is also a large gap between the number of patients with suspected OSA and the number of sleep laboratories capable of performing PSG resulting in long waiting lists. In addition questions about the effect of artificial environment of sleep laboratory on the measurement of disease severity have been raised (28). Elderly and/or sick patients may find the PSG too cumbersome and be reluctant to spend the night in the sleep laboratory. In 1992 Douglas et al $(29,30)$ reported no influence on their diagnostic conclusions when sleep parameters (i.e. $E E G, E O G \& E M G$ ) were excluded from the PSG evaluation of their patients, suggesting that devices which use only respiratory parameters may be useful in the evaluation of patients suspected of OSA. The use of such devices could provide a quick diagnosis to simpler cases and reduce the waiting time for more complicated cases. Portable monitoring (PM) has been proposed as a substitute for PSG in the diagnostic assessment of patients with suspected OSA. Compared to PSG, portable sleep studies require less technical expertise, are less labour-intensive and costly, and record patients in the natural environments of their own bed. Theterm portable monitoring encompasses a wide range of devices that can record as many physiological parameters as the standard PSG or only one parameter such as oximetry.

In 1994, American Academy of Sleep M edicine, (AASM , formerly the American Sleep Disorders Association) (31), categorized portable monitoring devices into four types: type I, standard polysomnography which was considered the reference standard to which other monitoring types were compared; type 2, comprehensive, unattended, portable polysomnography using the same bioelectrical signals as standard PSG ; type 3, modified portable sleep testing (also referred to as cardio respiratory sleep studies) which lack the bioelectrical signals for sleep staging and use a minimum of 4 channels, including ventilation (at-least 2 channels of respiratory movement, or respiratory movement or airflow), heart rate or ECG, oxygen saturation; type 4, continuous singleor dual bioparameter recording which employ minimum of one channel, i.e. oxygen saturation, flow or chest movement. When EEG and EM G signals are used, total sleep time (TST) can be calculated, that provides denominator for the calculation of $\mathrm{AHI}$ (number of events/hour of sleep). M ore commonly EEG \& EMG signals are not recorded by portable monitors, in which case the severity of breathing events is quantified per hour of recording time as respiratory disturbance index (RDI). Because of its larger denominator RD I would necessarily underestimate AH I to some degree. M ost of the type 4 monitors are usually oximetry based and therefore use oxygen saturation as the primary parameter to define breathing disturbances. Different methods of automatic analysis of oxygen saturation include a drop in oxygen saturation from $2 \%$ to $5 \%$, slope of resaturation curve or a combination of both criterias $(32,33)$. Some oximetry based monitors use cumulative percentage of time that oxygen saturation is below $90 \%$ i.e. $\mathrm{CT}_{90}$ as a criteria for diagnosis of sleep apnea. A CT ${ }_{90}$ of more than $1 \%$ is considered diagnostic of OSA (34). The delta index, which is a measure of variability in oxygen saturation over constant time intervals has also been used for the diagnosis of OSA (35). Recently one type 4 monitor used combined index of heart rate variability and oximetry (36) while another used flow sensors (oral and nasal thermistor) with real-time analysis hardware and software and miniature display unit (SleepStrip ${ }^{\mathrm{TM}}$ ) (37) for screening OSA.

There is no consensus about the ideal way for interpreting the data from portable monitors. Some systems use automatic analysis to detect and count events but may fail to identify poor quality recordings and thus can give misleading results. 0 thers depend on manual review by a sleep technician or physician, which raises the issue of inter and intra observer variability. Still others score events automatically but also have the provision for manual validation (40).

Several statistical methods have been used for evaluating the extent of agreement between the results of two diagnostic tests (AH I from PSG and RDI from a portable monitor). These include Pearson correlation coefficient, intra class correlation co-efficient, the approach of Bland and Altman of mean difference and limits of agreement and sensitivity/specificity/likelihood ratios(LRs). Although the Pearsen correlation coefficient is most commonly used, it is not recommended, because it is a measure of association not agreement (38). O n the other hand intra class correlation coefficient can be used to assess agreement but is not commonly used due to its non-familiarity with the clinicians (39). In the Bland and Altman method the difference between the measurements is calculated and then plotted against the mean of two numbers. The limit of agreement (i.e. the 
mean +2 SD of difference), which is the key descriptor that relates how well the two measures agree, if not calculated properly, can bemisleading (38). Thestatistical methods defining agreements between two tests, do not provide sufficient information to the clinician to decide whether a test correctly classifies patients as having or not having sleep apnea. For this sensitivity, specificity and likelihood ratios seem to be more useful. This approach uses an arbitrary cutoff for the $\mathrm{AHI}$ that is variable across the studies to classify a patient with or without sleep apnea. A receiver operative characteristic (ROC) curvefor an individual study will display the effect of changing diagnostic cutoff values of AHI upon the sensitivity and specificity of the test. A summary ROC curve in effect combines theindividual study RO C curves in a meta-analytic framework to give an overall picture of the diagnostic accuracy of a test over the range of cutoff values represented (46). The analysis of results using sensitivity, specificity and LRs should take into account the calculation of confidence intervals, which are a direct reflection of sample size and study design. Sensitivity, specificity and LRS are indicators of the operative characteristics of a test i.e. the degree to which the probability of disease is changed by a positive or a negative result. The likelihood ratio gives the most important information regarding the utility of a test. The LR for a positive test result is the ratio of the proportion of patients with disease who have a positive test (sensitivity) to the proportion of people without disease who have a positive test (false positive rate), whereas LR for a negative test result is the ratio of the proportion of patient with disease who have a negative test (false negative rate) to the proportion of people without disease who have a negative test (specificity). A high LR value for a positive test is useful for "ruling in" and a low LR value for a negative test is useful for "ruling out" sleep apnea. H owever, since a clinician needs to know the actual probability that the patient does or does not have a disorder (i.e., the post test probability), the operative characteristics of a test have to be interpreted with the knowledge of the pretest probability (or prevalence) of the disorder. This process can be simplified with the use of a normogram which highlights the interaction between pretest probability and LR on post test probability (40).

The use of portable monitoring to evaluate patients suspected of having OSA has been the subject of many reviews of literature in the last one and a half decade. Besides these reviews, guidelines and practice parameters were issued by a number of authors, including AASM and the Agency of $\mathrm{H}$ ealth $\mathrm{C}$ are Research and Q uantity (AH RQ)

In 1994 AASM practice parameters recommended unattended portable recording as an acceptablealternative to PSG only under thefollowing circumstances: (a) when initiation of treatment was urgent and PSG was unavailable, (b) when patient could not undergo PSG due to mobility issue and (c) as a follow up to treatment study. The use of type 4 monitor was not considered acceptable for the diagnosis of OSA $(41,42)$. In 1997, the AASM published another review (43) and practice parameter (44) for PSG and related procedures that included a section on Type 3 and 4 monitors. Thepractice parameters recommended that attended Type 3 monitors were potentially appropriate in patients with high probability (e.g., $>70 \%$ ) of sleep apnea and that negative type 3 monitor studies in symptomatic patients should be followed up with a full PSG. The parameters did not recommend type 4 studies for the investigation of suspected OSA. Also in 1997, the Agency of $\mathrm{H}$ ealth C are Research and Q uantity (AHRQ) carried out a systematic review of the research on the diagnosis of OSA $(45,46)$. Part of this review was focused on the portable monitors (25 studies). The quality of each reviewed study was rated using a scale developed by the authors, which attempted to identify and account for biases that may undermine the validity of findings and conclusion of a study. The authors of this review concluded that there was some evidence in a relatively small number of patients that should be expanded with more studies, suggesting that a full laboratory PSG may not be necessary to diagnose sleep apnea. Rather, attended in laboratory partial channel PSG in the context of high likelihood of OSA based upon clinical features have significantly high sensitivity ( $82 \%$ to $94 \%$ ) and specificity $(82 \%$ to $100 \%)$ to replace full PSG. There was still insufficient evidence that any multi-channel portable device could be used in the home settings $(45,46)$. Sensitivity of portable devices ranged from $78 \%$ to $100 \%$ and specificity ranged from $62 \%$ to $94.5 \%$. It was also observed that in general, the diversity of study designs and objectives were very high and the methodological rigor of these studies as assessment of diagnostic tests was very low. The authors recommended that future research should include standardization of terms and diagnostic criteria, and consistently reported statistics to enhance the utility of published literature $(45,46)$.

Indian Journal of Sleep M edicine (IJSM ), Vol. 1, N o. 2, 2006 
In a recent review based on 49 articles in the peerreviewed literature published by AASM in collaboration with American Thoracic Society (ATS) and American College of Chest Physicians (ACCP)(47), the authors selected three primary and four secondary end points while analysing the published results. The primary endpoints included the ability of portable devices to reduce the probability that a patient has an abnormal $\mathrm{AHI}$ (to rule out the disorder), increase the probability that a patient has an abnormal $\mathrm{AHI}$ (to rule in the disorder), and both reduce and increase the probability that a patient has an abnormal AH I (to both rule out and rule in the disorder). The authors also reviewed secondary end points, including the reproducibility of portablemonitors results, the cost and benefit of using of portable devices, the failure rates of portable devices, patient populations studied, and the generalizability of findings. A meta analysis of results was not used because too much heterogeneity existed between studies with respect to types of signals measured, criteria used to define a breathing event, scoring of signals from portable devices, and study quality. The articles were rated using the method published by Sackett et al (48) in 2000 for rating evidence of research on diagnostic test because it closely aligns with the accepted methods used for rating the quality of articles on therapeutics and prognosis. In addition this method focused on the key aspects of the design of studies that are used to evaluate the diagnostic test: avoiding selection bias (by using a consecutively referred sample of patients), verification bias (by performing the reference standard on all subjects), and blinding interpreters.

Based on the data from this review article AASM developed practice parameters (49) regarding the use of portable monitors for the diagnosis of suspected OSA. These are as follows: (a) insufficient evidence is available to recommend the use of type 2 devices in attended or unattended settings; (b) type 3 devices appear to be capable of being used in an attended setting to increase or to decrease the probability that a patient has an $\mathrm{AH}$ I of $>15$, and may rule in or rule out OSA when conducted on suitable patients and interpreted by manual scoring by trained personnel. Appropriate patients for this use should be free from significant co-morbid conditions, and symptomatic patients with negative portable sleep studies should undergo attended PSG to truly exclude OSA; (c) the use of type 3 sleep studies in an unattended settings is not recommended at this time; (d) the use of type 4 devices was not recommended in attended or unattended settings for diagnosis of OSA.

It was also recommended that portable devices should not be used for general population screening, in the absence of a pretest probability of the patient having a diagnosis of OSA and for complaints other than those associated with OSA. In addition they should not be used without review of raw data during interpretation, by physicians without familiarity with their use and limitation, and without trained personnel to perform technical scoring (49).

Several shortcomings were identified in the published studies which resulted in poor validation of the findings. All studies had taken place on patients referred to sleep centres with high pretest probability of sleep apnea leading to increased number of false negative results. Primary care populations, women, patients with comorbid illness such as heart failure and chronic obstructive pulmonary disease, and ethnic populations other than whites were not studied adequately. In general, studies included small sample size and were not particularly well-designed. The findings of any onestudy were integrally limited, meaning thereby that results obtained for a particular device were applicable only to that device and could not be extrapolated to other devices. Even within a given device class, (e.g. oximetry) results may be affected by the data-processing methods, including digital signal analysis, sampling rate and averaging time. The quality of the studies also varied widely. Common reasons for having a lower quality rating included poor description of the device used, lack of definition of respiratory events, lack of description of blinding and inability to perform the manual validation of the automatic analysis. The majority of studies having a high level of evidenceand high quality rating on portable monitors had been performed in the attended setting and data proving cost effectiveness of portablemonitoring were lacking. The role of unattended portable monitoring, therefore, was not yet fully established (47).

The main method used for assessing the validity of a portable monitor is by comparing its RDI with the simultaneously measured AH I from standard PSG using an arbitrary cut-off value of $\mathrm{AHI}$ for the diagnosis of OSA. This value is variable across the studies. This method may be misleading in clinical practice, as both PSG and portable monitors have considerable night-tonight variation and even a minor difference between RD I from portable monitor and $\mathrm{AH}$ I from PSG, if this difference crosses the arbitrary cut off value, can 
significantly affect the sensitivity and specificity. There is no statistical or practical difference between patients having $\mathrm{AHI}$ few points above or below a threshold. M oreover, AH I by itself has limited clinical significance, correlating poorly with symptoms or with outcome of treatment $(50,51)$. So there is a need to evaluate portable monitors from a different perspective, such as decision to treat or predicting the outcome of treatment.

Recently a few studies of unattended type 3 portable monitoring in a home setting have been published. In a study by Zou et al (52), portable monitoring (using peripheral arterial tone, heart rate, actigraphy) was reasonably accurate for home diagnosis of OSA in a population sample not preselected for OSA symptoms. The accuracy of the portable monitor was assessed by comparison with data from simultaneous unattended home PSG recordings. Yin et al (53) evaluated the reliability of type 3 portablemonitor in unattended home setting and found a high sensitivity in general but a low specificity in patients with mild disease. The accuracy of the result was affected by $\mathrm{AHI}$, recording time and sleep position. In an another study by the same author (54), unattended home monitoring was found useful provided the data analysis was performed manually. Whitelaw et al,(55) evaluated an oximetry based home monitoring system and found it useful to measure the accuracy with which sleep physicians can predict which patients would ben efit from treatment of OSA. It was found that the ability of physicians to predict the outcome of nasal CPAP was not significantly better with PSG than with home oximetry based monitor. Recently a new method i.e. Apnea risk evaluation system (ARES) was evaluated in the unattended home setting with high sensitivity and specificity for the diagnosis of OSA (56).

The role of unattended portable monitoring in the diagnosis of OSA is continuously evolving with development of new technologies, study designs and strategies for application. The technology needs to be sound and reliable. The study designs should include the cost benefit analysis, stratification of patients, and the prediction of response to treatment (57). The role of homemonitoring should al so be assessed in the context of diagnostic and treatment algorithms and final patient outcomes. There is a strong need to carry out similar studies in the Indian scenario, taking into account specific local factors which can affect the validity of portable monitoring, such as level of education, socio-economic status, frequent power failures / breakdowns and cultural traditions. After the availability of this information, the actual role of unattended home monitoring will be determined for the diagnosis of OSA.

\section{Authors' affiliations}

Dr. J. C. Suri, Head of the Department, Department of Pulmonary, Critical Care \& Sleep Medicine, Vardhaman Mahavir Medical College \& Safdarjang Hospital, N ew Delhi

\section{References}

1. Guilleminault C, Tilkian A, Dement WC: The sleep apnea syndrome. Annu Rev Med 1976; 27:465-484.

2. Young T, Palta M, Dempsey J, et al. The occurrence of sleep-disordered breathing among middle-aged adults. N EnglJ Med 1993;328:1230-1235.

3. Mitter M. Daytime sleepiness and cognitive functioning in sleep apnea. Sleep 1993;16:S68-S70.

4. Kim H, Young T, Matthews C, Weber S, Woodward A, Palta $M$. sleep disordered breathing and neuropsychological deficits: a population based study. Am J Respir Crit Care Med 1997;156:1813-1819

5. Horstmann S, Hess CW, Bassetti C, gugger M, Mathis J. Sleep related accidents in sleep apnea patients. Sleep 2000;23:383-389.

6. Ulfberg J, Carter N, Talback M, Edling C. Excessive daytime sleepiness at work and subjective work performance in the general population and among heavy snorers and patients with obstructive sleep apnea. Chest 1996;10=10:659-663.

7. Lavie $\mathbf{P}$, Herer $\mathbf{P}$, Hoffstein V. Obstructive sleep apnoea syndrome as a risk factor for hypertension: population study. BMJ 2000;320:479-482.

8. Nieto FJ, Young TB, Lind BK, et al. Association of sleepdisordered breathing, sleep apnea, and hypertension in a large community-based study. Sleep Heart Health Study. JAMA 2000;283:1829-1836.

9. Peppard PE, Young T, Palta M, Skatrud J. Prospective Study of the association between sleep-disordered breathing and hypertension. N EnglJ med 2000;342:1378-1384

10. Bixler EO, Vgontzas AN, Lin HM, et al. Association of hypertension and sleep-disordered breathing. Arch Intern Med 2000;160:2289-2295.

11. Andreas S, Hajak G, Breska BV, Ruther E, Kreuzer H. Changes in heart rate during obstructive sleep apnoea. Eur RespirJ 1992;5:853-857.

12. Koskenvuo $\mathbf{M}$, Kaprio J, Telvakivi T, partinen M, Heikkila K, Sarna S. Snoring as a risk factor for ischemic heart disease and stroke in men. BMJ 1987;294:16-19.

13. Andreas S, Schultz R, Werner GS, Kreuzer H. Prevalence of obstructive sleep apnea with coronary artery disease. Chest 1996;7:541-545.

14. He J, Kryger MH, Zorik FJ, Conway W, Roth T. Mortality and apnoea index in obstructive sleep apnea. Chest 1988;94:914

15. Lavie $\mathbf{P}$, Herer $\mathbf{P}$, Peled $\mathrm{R}$, et al. Mortality in sleep apnea

Indian Journal of Sleep M edicine (IJSM ), Vol. 1, N o. 2, 2006 
patients: a multivariate analysis of risk factors. Sleep 1995;18:149-157.

16. Dyken ME, Somers VK, Yamada T, Ren ZY, Zimmerman $M B$. Investigating the relationship between stroke and obstructive sleep apnea. Stroke 1996;27:401-407.

17. Phillipson EA. Sleep apnea-a major public health problem (Editorial). N Engl J Med 1993;328:1271-3

18. Teran-Santos J, J imenez-Gomes, A, Cordero-Guevara J, The Cooperative Group Burgos-Santander. The association between sleep apnea and the risk of traffic accidents. N EnglJ Med 1999;340:847-51

19. Pack Al, Maislin G, Staley B, George C, Pack FM, Dinges DF. Factors associated with daytime sleepiness and performance in a sample of commercial drivers [abstract]. Sleep 2001;24:A427.

20. Sullivan C, Issa F, Berthon-Jones M, Eves L. Reversal of obstructive sleep apnea by continuous positive airways pressure applied through the nares. Lancet 1982;1:862865.

21. Jenkinson C, Davies RJ, Mullins R, Stradling JR. Comparision of therapeutic and subtherapeutic continuous positive airway pressure for obstructive sleep apnoea: a randomized prospective parallel trial. Lancet 1999;353:21002105.

22. Heinrich F Becker, Andreas Jerrentrup, Thomas Ploch, Ludger Grote, Thomas Penzel, Colin E. Sullivan, and J. Hermann Peter. Effect of Nasal Continuous Positive Airway Pressure Treatment on Blood Pressure in Patients With O bstructive Sleep Apnea. Circulation, Jan 2003; 107: 68 73.

23. Barnes M, Houston D, Worsnop C, N eill A, Mykytyn I, Kay A. Trinder J, Sauders N, McEvoy D, Pierce R. A randomized controlled trial of continuous positive airway pressure in mild obstructive sleep apnoea. Thorax 2000;55:224-231.

24. Engleman H, Kingshott R, Wraith P, Mackay T, Deary I, Douglas N. Randomized placebo-controleld crossover trial of continuous positive airway pressure for mild sleep apnea/ hypopnea syndrome. Am J Respir Crit Care Med 1999;159:461-467.

25. Redline S, Adams N, Strauss M, Roebuck T, Winters M, Rosenberg C. Improvement of mild sleep-disordered breathing with CPAP compared with conservative therapy. Am J Rspir Crit Care Med 1998;157:858-865.

26. Collision MO, Man GCW, Kang BV. Level of awareness of sleep apnea among Alberts's physicians: a survey. Sleep Res 1995;24:214

27. AASM Task Force. Sleep-related breathing disorders in adults: recommendations for syndrome definition and measurement techniques in clinical research. Sleep 1999;22:667-89.

28. Lavie P. Incidence of sleep apnea in a presumably healthy working population: a significant relationship with excessive daytime sleepiness. Sleep 1983;6:312-18.

29. Douglas NJ, Thomas S, Jan MA. Clinical value of polysomnography. Lancet 1992;339:347-50 30. K. Dingli, E.L. Coleman, M. Vennelle, S.P. Finch, P.K. Wraith, T. . Mackay, N.J. Douglas. Evaluation of a portable device or diagnosing the sleep apnea/hypopnoea syndrome. Eur Respir J 2003; 21: 253-259.

31. Ferber R, Millman R, Coppola M, Fleetham J, Murray $C F$, Iber $C$, et al. portable recording in the assessment of obstructive sleep apnea. Sleep 1994;17:378-92.

32. Rauscher $\mathbf{H}$, Popp W, Zwick H. Model for investigating snorers with suspected sleep apnea. Thorax 1993;48:2759

33. Vazquez J, Tsai WH, Flemons WW, Masuda A, Brant R, Hajduk $E$, et al. Automated analysis of digital oximetry in the diagnosis of obstructive sleep apnoea. Thorax 2000;55:302-7.

34. Gyulay S, O Ison LG, Hensley MJ, King MT, Murree Allen K, Saunders NA. A comparison of clinical assessment and home oximetry in the diagnosis of obstructive sleep apnea. Am Rev Respir Dis 1993;147:50-3.

35. Pepin J L, Levy P, Lepaulle B, Brambilla C, Guilleminault C Does oximetry contribute to the detection of apneic events? Mathematical processing of the $\mathrm{SaO}_{2}$ signal. Chest 1991;99:1151-7

36. Ben Raymond, R. M. Cayton, M. J. Chappell. Combined index of heart rate variability and oximetry in screening for the sleep apnoea/hypopnoea syndrome. J. Sleep Res. 2003;12:53-61.

37. T. Shochat, N. Hadas, M. Kerkhofs, A. Herchuelz, T. Penzel, J.H.Peter, P. Lavie. The SleepStrip ${ }^{\mathrm{TM}}$ : an apnoea screener for the early detection of sleep apnoea syndrome. Eur Respir J 2002;19:121-126.

38. Bland J M, Alltman DG. Statistical methods for assessing agreement between two methods of clinical measurement. Lancet 1986;1:307-10.

39. Streiner DL, N orman GB. Health measurement scales: a practical guide to their development. $2^{\text {nd }}$ ed. New York, NY: Oxford University Press, 1995.

40. Christopher K. Li, Flemons W.W. State of home sleep studies. Clin Chest Med 2003; 24:283-295.

41. Standards of Practice Committee of the American Sleep Disorders Association. Practice parameters for the use of portable recording in the assessment of obstructive sleep apnea. Sleep 1994;17:372-7.

42. Ferber RA, Mullman RP, coppola MP, et al. ASDA standards of practice: portable recording in the assessment of obstructive sleep apnea. Sleep 1994;17:378-392.

43. Chesson AL, Ferber RA, Fry JM, et al. The indications for polysomnography and related procedures. Sleep 1997;20:423-487.

44. American Sleep Disorders Association. Practice parameters for the indications for polysomnography and related procedures: Polysomnography Task force, American sleep Disorders Association Standards of practice Committee. Sleep 1997;20:406-422.

45. Ross SD, Allen IE, Harrison KJ, et al. Systematic review of the literature regarding the diagnosis of sleep apnea: evidence report/technology assessment No.1. Rockville, MD: Agency for Health Care Policy and Research; February 1999; AHCPR Publication N 0.99-002. 
46. Ross SD, Sheinhait IA, Harrison KJ et al. Systematic review and meta-analysis of the literature regarding the diagnosis of sleep apnea. Sleep 200;23:519-532.

47. Flemons $\mathbf{W}$, Littner $\mathbf{M}$, Rowley J, et al. Home diagnosis of sleep apnea: a systematic review of the literature; and evidence review cosponsored by the American Academy of Sleep Medicine, the American college of Chest Physicians, and the American Thoracic Society. Chest 2003;124:15431579.

48. Sackett D. Rules of evidence and clinical recommendations for the management of patients. Can J Cardiol 1993;9:4879.

49. Chesson A, Berry R, Pack A. Practice parameters for the use of portable monitoring devices in the investigation of suspected obstructive sleep apnea in adults. Sleep 2003;26:1-7

50. Adams N, Strauss M, Schluchter M, Redline, S. Relation of measures of sleep-disordered breathing to neuropsychological functioning. Am J Respir Crit Care Med 2001;163:1626-1631.

51. Flemons $\mathbf{W} \mathbf{W}$, Reimer MA. Measurement properties of the Calgary Sleep Apnea Q uality of Life Index. Am J Respir Crit Care Med 2002;165:159-164.

52. Zou D, Grote L, peker Y, Lindblad U, Hedner]. Validation a portable monitoring device for sleep apnea diagnosis in a population based cohort using synchronized home polysomnography. Sleep 2006 Mar 1;29(3):367-74.

53. Yin M, Miyazaki S, Ishikawa K. Evaluation of type 3 portable monitoring in unattended home setting for suspected sleep apnea: factors that may affect its accuracy. O tolaryngol Head Neck surg. 2006 Feb;134(2):204-9.

54. Yin M, Miyazaki S, Itasaka Y, Shibata Y, Abe T, Miyoshi A, Ishikawa K, Togawa K. A preliminary study on application of portable monitoring for diagnosis of obstructive sleep apnea. Auris N asus Larynx. 2005 J un;32(2):151-6.

55. William A. Whitelaw, Rollin F. Brant and W. Ward Flemons. Clinical Usefulness of Home Oximetry Compared with Polysomnography for Assessment of Sleep Apnea. Am J Respir Crit Care Med Vol 171. pp 188-193, 2005

56. Philip R. Westbrook, Daniel J. Levendowski, Milenko Cvetinovic, Timothy Zavora, Vladislav Velimirovic, Delmer Henninger, Dennis Nicholson. Description and validation of the apnea risk evaluation system: a novel method to diagnose sleep apnea-hypopnea in the home. Chest 0 ctober 2005; Volume 128 - Number 4

57. Collop N.A. Portable monitoring for diagnosing obstructive sleep apnea, not yet ready for primetime. Chest 2004;125:3:809-810. 